\title{
Editorial
}

Peter B. Luppa

\section{Point-of-care testing at the interface of emerging technologies and new clinical applications}

https://doi.org/10.1515/labmed-2020-0020

Point-of-care testing (POCT) enables health care professionals to perform laboratory medicine tests near the patient. The underlying technology and the range of test parameters available are evolving rapidly. Today, in many clinical processes, POCT is used routinely, shortening the time for clinical decision-making about additional testing or therapy, as delays are no longer caused by preparation of clinical samples, transport, and central laboratory analysis. It is worth mentioning that POCT and central laboratory together are important for the optimal functioning of diagnostic processes. They complement each other, provided that a dedicated POCT team integrates the quality assurance of POCT into the overall quality management system of the hospital.

The theme of the $4^{\text {th }}$ Munich POCT Symposium 2019 as mentioned in the headline - can be seen as a kaleidoscope focusing on the emerging fascinating analytical techniques as well as on evidence-based applications of POCT methods. The conference was also bridge building to relevant questions regarding economics and medical care. In this issue of the Journal of Laboratory Medicine, a number of current hot topics of POCT discussed during the symposium will be presented to further support the rational development and application of the patient-near testing. The first two articles from Bietenbeck and Luppa [1], as well as from Kachler and Maschek [2] give detailed information on the 10 different sessions, held at the 3-day meeting.

Five thematic areas concerning POCT were of outstanding interest: emergency medicine, chronic disease management, emerging analytical technologies, quality assurance, and data security.

The first issue of POCT in emergency medicine is discussed in an article by Collinson, with special emphasis on cardiac biomarkers in emergency and intensive care medicine [3]. He describes, on one side, the changing management strategies in patients with acute chest pain. The diagnosis of acute myocardial infarction (AMI) in patients with chest pain has undergone a series of step changes. First, the medical management was supportive, and active intervention was considered but not universally accepted. But the demonstration of opening the coronary arteries by thrombolysis and by acute catheterization led to a reappraisal. This strategy has become the standard of care, and catheterization and coronary stenting are stateof-the-art nowadays. Due to the fact that the majority of patients with chest pain did not have AMI, rapid strategies were developed in order to make earlier identification of patients with AMI possible (rule-in) but also to identify patients without AMI (rule-out). Diagnostic strategies based on rapid serial measurements of myoglobin, creatine kinase (CK), and CK-myocardial band (CK-MB) were developed including the concept of delta change. Thus, measurement of cardiac biomarkers shifted from ancillary test to an integral part of the strategy of cardiac patient assessment. In the second part of the article, Collinson also portrays the role of POCT in the era of highly sensitive cardiac troponin (hs-cTn) assays. According to the latest European Society of Cardiology (ESC) guideline, repeated measurements of hs-cTn initially and $1-2 \mathrm{~h}$ after admission, performed in the central laboratory (CL), is best practice. However, this service cannot be offered by many laboratories. Therefore, POCT measurements would be helpful and early studies suggest that it is the impact of having results within the timeframe of the patient consultation that triggers the clinical decisions. Thus, the provision of hs-cTn measurement by POCT would be the ideal tool for diagnostic algorithms. However, this is not yet met by almost all of the commercially available POCT systems. Comparable diagnostic sensitivity to hs-cTn measured in the CL is not achieved for POCT until typically $6 \mathrm{~h}$ from admission.

Chronic diseases pose major burden to patients and health care systems. The conference enabled here an indepth discussion on POCT applications and how patient self-testing could contribute to the management of chronic disease. In his article [4], O'Kane states that there is universal agreement that patients with diabetes treated with insulin, regardless of the type of diabetes, should be offered the possibility of undertaking capillary glucose monitoring. Other examples of chronic diseases are also given by the author: use of POCT for anticoagulant 
monitoring and renal function monitoring after kidney transplantation.

A theoretical framework is worth considering: Selftesting by the patient is only of value if it can be translated into benefit. Possible benefits in this context include improved clinical outcomes, improvement in surrogate markers, such as HbA1c in diabetics, greater convenience or improved psychological well-being. These benefits may also contribute to reduced costs of care. Several theoretical criteria must be met if self-testing is to deliver benefit: Firstly, the test must provide useful information on the patient's condition that leads to direct treatment decisions. Secondly, the analytical performance of the POCT self-monitoring test should be appropriate to the clinical application. Thirdly, self-testing can only be undertaken by a supported, engaged, and informed patient who is also willing to take appropriate action on the results obtained.

Most vibrating novel POCT applications are nucleic acid testing (NAT) methods for the identification of infectious agents and continuous glucose monitoring (CGM) devices for diabetics. Here, emerging technologies actually meet clinical needs. It must be emphasized that both technologies are becoming increasingly common in hospitals and outpatient centers, while meaningful quality assurance regularities are lagging behind.

Among the currently available patient-near laboratory diagnostic procedures, methods for the diagnosis of infectious agents play a special role. Besides systems based on the immunochromatographic detection of a specific microbial antigen, there are now a variety of molecular biological methods for the diagnostic amplification of the pathogen DNA/RNA. For clinical chemistry POCT devices, a series of different international quality standards and guidelines are available regarding their implementation and quality assessment. However, these rules tend to lag behind for dedicated NAT-POCT devices. It is obvious for CGM that measures for accuracy and precision are not yet depicted sufficiently.

To shed light on the field of CGM, Freckmann [5] gives an overview on currently available CGM systems which measure glucose levels in the subcutaneous interstitial fluid for 6-14 days. In the section "sensor metrics and accuracy", the author presents new approaches for the quality assessment of CGM. Alarm and trend arrow functionalities as well as a clear and intuitive data presentation encourage the self-management of diabetes therapy. The high frequency of glucose data and the multitude of existing functions require an extensive training of diabetics (diabetes type 1 [T1D] and type 2 [T2D]). On the other hand, there are already convincing clinical studies available demonstrating the benefits of CGM systems in diabetes therapy in terms of a decreasing number of hypoglycemic events, improvement of glycemic control, and significant reduction of HbA1c in diabetics. The German HypoDE trial, a 6-month, multicenter study with T1D patients with impaired hypoglycemia awareness or severe hypoglycemia showed a significantly reduced incidence of hypoglycemia events by $72 \%$ when using CGM compared to capillary glucose measurements. Other studies also demonstrate that CGM is positively correlated with the time period spent in euglycemia and inversely associated with the time spent in hypo- or hyperglycemia. In summary, CGM systems provide a much more detailed picture of the glycemia 24-h profile in diabetes patients. In this way, educated diabetics are enabled to use these data to react adequately to their glucose levels and subsequently to avoid hypoglycemic and hyperglycemic events in the long term.

Another important and permanent issue of POCT management is quality assurance (QA). Here the article of von Meyer et al. [6] gives new interesting insights for the use of supplementary POCT quality controls (QC). This new "extended internal QC" uses in-house-generated QC material from left-over full blood from routine diagnostics. The performed study shows that measurements of quality control material beyond the mandatory QA plan can be structured and optimized by the use of a special POCT middleware solution.

The last of the thematic areas with outstanding interest is data security in POCT applications.

Johannis et al. [7] develop new IT security concepts in their contribution. In Germany and in many other European countries, hospital IT-security regulations have not yet been adapted to POCT. The authors report also on the " 1 st Round Table on POCT IT-Security", which was held in Cologne, October 2019, which followed the first discussions during the $4^{\text {th }}$ Munich POCT symposium. The authors report first consensus results in the fields of user-, data-, and update management of POCT devices and POCT network connections. They suggest that the first steps should include the optimization of the POCT user management by connection to a directory service and definition of access control (including emergency authorization). Patient data economy on the devices in combination with data and data transmission encryption together with secure communication protocols are relevant in this context. Periodically, updates need to be contractually defined for remote services and include testing in a protocol-based scenario. Hospitals with a 
competent POCT coordination should provide organizational structures for IT security and are responsible for continuous training (e.g. in the form of e-learning). Periodic evaluations of the system are indicated to achieve the goal of a secure POCT IT network as a part of the hospital IT security.

In summary, the articles collated in this special issue of the Journal of Laboratory Medicine highlight the importance of POCT technology for diagnostic processes and give the evidence base relating to the applications in different clinical fields. These range from the emergency department to patient self-testing in diabetes and other diseases. The contributions also deal with questions related to economics, medical care, and patient safety and provide viable solutions.

Author contributions: The author has accepted responsibility for the entire content of this submitted manuscript and approved submission.

Research funding: None declared.

Employment or leadership: None declared.

Honorarium: None declared.

\section{References}

1. Bietenbeck A, Luppa PB. Congress report: 4th Munich POCT Symposium 2019, March 11-13, 2019, Klinikum rechts der Isar der TU München. J Lab Med 2020;44:63-70.

2. Kachler M, Maschek C. Organizational challenges in the management of point-of-care diagnostics in healthcare facilities. J Lab Med 2020;44:103-5.

3. Collinson P. Cardiac biomarkers by point-of-care testing - back to the future? J Lab Med 2020;44:89-95.

4. O'Kane MJ. Patient self-testing in chronic disease management. J Lab Med 2020;44:81-7.

5. Freckmann G. Basics and use of continuous glucose monitoring (CGM) in diabetes therapy. J Lab Med 2020;44:71-9.

6. von Meyer A, Luppa PB, Cadamuro J. The extended internal QC (eIQC): a new practical approach for quality assurance in point-ofcare glucose testing using the POCTopus Software - a pilot study. J Lab Med 2020;44:97-102.

7. Johannis W, Bietenbeck A, Malchau G, Streichert T. Point-of-care testing (POCT) and IT security concepts. J Lab Med 2020;44:107-11.

Correspondence: Peter B. Luppa, Klinikum rechts der Isar der Technischen Universitaet Muenchen - Institut fuer Klinische Chemie und Pathobiochemie, Ismaninger Str. 22, Munich 81675, Germany, E-Mail: p.luppa@tum.de 